JOURNAL OF THE

AMERICAN MATHEMATICAL SOCIETY

Volume 15, Number 3, Pages 665-670

S 0894-0347(02)00394-6

Article electronically published on February 27, 2002

\title{
GROWTH OF SOLUTIONS FOR QG AND 2D EULER EQUATIONS
}

\author{
DIEGO CORDOBA AND CHARLES FEFFERMAN
}

\section{INTRODUCTION}

The work of Constantin-Majda-Tabak [1] developed an analogy between the Quasi-geostrophic and 3D Euler equations. Constantin, Majda and Tabak proposed a candidate for a singularity for the Quasi-geostrophic equation. Their numerics showed evidence of a blow-up for a particular initial data, where the level sets of the temperature contain a hyperbolic saddle. The arms of the saddle tend to close in finite time, producing a sharp front. Numerics studies done later by Ohkitani-Yamada [8] and Constantin-Nie-Schorghofer [2], with the same initial data, suggested that instead of a singularity the derivatives of the temperature were increasing as double exponential in time.

The study of collapse on a curve was first studied in [1] for the Quasi-geostrophic equation where they considered a simplified ansatz for classical frontogenesis with trivial topology. At the time of collapse, the scalar $\theta$ is discontinuous across the curve $x_{2}=f\left(x_{1}\right)$ with different limiting values for the temperature on each side of the front. They show that under this topology the directional field remains smooth up to the collapse, which contradicts the following theorem proven in [1]:

If locally the direction field remains smooth as $t$ approaches $T_{*}$, then no finite singularity is possible as $t$ approaches $T_{*}$.

The simplified ansatz with trivial topology studied in [1] does not describe a hyperbolic saddle.

Under the definition of a simple hyperbolic saddle, in [3], it was shown that the angle of the saddle cannot decrease faster than a double exponential in time.

The criterion obtained in [5] for a sharp front formation for a general twodimensional incompressible flow is:

A necessary condition to have a sharp front at time $T$ is

$$
\int_{0}^{T}|u|_{L^{\infty}}(s) d s=\infty
$$

Received by the editors March 25, 2001.

1991 Mathematics Subject Classification. Primary 76B03, 35Q30; Secondary 35Q35, 76W05.

Key words and phrases. Quasi-geostrophic, Euler and MHD equations, front formation, singularities.

This work was initially supported by the American Institute of Mathematics.

The second author was supported in part by NSF Grant DMS 0070692. 
For the Quasi-geostrophic equation it is not known if the quantity $\int_{0}^{T}|u|_{L^{\infty}}(s) d s$ diverges or not, and the criterion does not say how fast the arms of a saddle can close.

In this paper we do not assume anything on the velocity field, and we show that under a semi-uniform collapse the distance between two level curves cannot decrease faster than a double exponential in time. The semi-uniform collapse assumption greatly weakens the assumptions made in [1] for an ansatz for classical frontogenesis, and the simple hyperbolic saddle in 3 .

In the case of $2 \mathrm{D}$ incompressible Euler equations we are interested in the large time behavior of solutions.

The two equations we discuss in this paper have in common the property that a scalar function is convected by the flow, which implies that the level curves are transported by the flow. The possible singular scenario is due to level curves approaching each other very fast which will lead to a fast growth on the gradient of the scalar function. Below we study the semi-uniform collapse of two level sets on a curve. By semi-uniform collapse we mean that the distance of the two curves at any point are comparable.

The equations we study are as follows:

\section{The Quasi-geostrophic (QG) Equation}

Here the unknowns are a scalar $\theta(x, t)$ and a velocity field $u(x, t)=\left(u_{1}(x, t)\right.$, $\left.u_{2}(x, t)\right) \in R^{2}$, defined for $t \in\left[0, T^{*}\right)$ with $T^{*} \leq \infty$, and for $x \in \Omega$ where $\Omega=R^{2}$ or $R^{2} / Z^{2}$. The equations for $\theta, u$ are as follows:

$$
\begin{array}{r}
\left(\partial_{t}+u \cdot \nabla_{x}\right) \theta=0, \\
u=\nabla_{x}^{\perp} \psi \text { and } \psi=\left(-\triangle_{x}\right)^{-\frac{1}{2}} \theta,
\end{array}
$$

where $\nabla_{x}^{\perp} f=\left(-\frac{\partial f}{\partial x_{2}}, \frac{\partial f}{\partial x_{1}}\right)$ for scalar functions $f$. The initial condition is $\theta(x, 0)=$ $\theta_{0}(x)$ for a smooth initial datum $\theta_{0}$.

\section{The Tho-Dimensional Euler Equation}

The unknown is an incompressible velocity field $u(x, t)$ as above with vorticity denoted by $\omega$. The $2 \mathrm{D}$ Euler equation may be written in the form

$$
\begin{array}{r}
\left(\partial_{t}+u \cdot \nabla_{x}\right) \omega=0, \\
u=\nabla_{x}^{\perp} \psi \text { and } \psi=\left(-\triangle_{x}\right)^{-1} \omega,
\end{array}
$$

with $u(x, 0)$ equal to a given smooth divergence free $u_{0}(x)$.

\section{Results}

Asssume that $q=q(x, t)$ is a solution to (1.1) or (1.2), and that a level curve of $q$ can be parameterized by

$$
x_{2}=\phi_{\rho}\left(x_{1}, t\right) \text { for } x_{1} \in[a, b]
$$

with $\phi_{\rho} \in C^{1}\left([a, b] \cap\left[0, T^{*}\right)\right)$, in the sense that

$$
q\left(x_{1}, \phi_{\rho}\left(x_{1}, t\right), t\right)=G(\rho) \text { for } x_{1} \in[a, b],
$$

and for certain $\rho$ to be specified below.

The stream function $\psi$ satisfies

$$
\nabla^{\perp} \psi=u .
$$


From (2.1) and (2.2), we have

$$
\begin{gathered}
\frac{\partial q}{\partial x_{1}}+\frac{\partial q}{\partial x_{2}} \frac{\partial \phi_{\rho}}{\partial x_{1}}=0 \\
\frac{\partial q}{\partial t}+\frac{\partial q}{\partial x_{2}} \frac{\partial \phi_{\rho}}{\partial t}=0
\end{gathered}
$$

By (1.1), (1.2), (2.3), (2.4) and (2.5) we obtain

$$
\begin{aligned}
\frac{\partial \phi_{\rho}}{\partial t} & =-\frac{\frac{\partial q}{\partial t}}{\frac{\partial q}{\partial x_{2}}}=\frac{\left\langle-\frac{\partial \psi}{\partial x_{2}}, \frac{\partial \psi}{\partial x_{1}}\right\rangle \cdot\left\langle\frac{\partial q}{\partial x_{1}}, \frac{\partial q}{\partial x_{2}}\right\rangle}{\frac{\partial q}{\partial x_{2}}} \\
& =\left\langle-\frac{\partial \psi}{\partial x_{2}}, \frac{\partial \psi}{\partial x_{1}}\right\rangle \cdot\left\langle\frac{\frac{\partial q}{\partial x_{1}}}{\frac{\partial q}{\partial x_{2}}}, 1\right\rangle \\
& =\left\langle-\frac{\partial \psi}{\partial x_{2}}, \frac{\partial \psi}{\partial x_{1}}\right\rangle \cdot\left\langle-\frac{\partial \phi_{\rho}}{\partial x_{1}}, 1\right\rangle .
\end{aligned}
$$

Next

$$
\begin{aligned}
\frac{\partial}{\partial x_{1}}\left(\psi\left(x_{1}, \phi_{\rho}\left(x_{1}, t\right), t\right)\right) & =\frac{\partial \psi}{\partial x_{1}}+\frac{\partial \psi}{\partial x_{2}} \frac{\partial \phi_{\rho}}{\partial x_{1}} \\
& =\left\langle-\frac{\partial \psi}{\partial x_{2}}, \frac{\partial \psi}{\partial x_{1}}\right\rangle \cdot\left\langle-\frac{\partial \phi_{\rho}}{\partial x_{1}}, 1\right\rangle
\end{aligned}
$$

Therefore

$$
\frac{\partial \phi_{\rho}}{\partial t}=\frac{\partial}{\partial x_{1}}\left(\psi\left(x_{1}, \phi_{\rho}\left(x_{1}, t\right), t\right)\right)
$$

With this formula we can write an explicit equation for the change of time of the area between two fixed points $a, b$ and two level curves $\left(\phi_{\rho_{1}}, \phi_{\rho_{2}}\right)$ :

$$
\begin{aligned}
& \frac{d}{d t}\left(\int_{a}^{b}\left[\phi_{\rho_{2}}\left(x_{1}, t\right)-\phi_{\rho_{1}}\left(x_{1}, t\right)\right] d x_{1}\right) \\
& =\psi\left(b, \phi_{\rho_{2}}(b, t), t\right)-\psi\left(a, \phi_{\rho_{2}}(a, t), t\right) \\
& +\psi\left(a, \phi_{\rho_{1}}(a, t), t\right)-\psi\left(b, \phi_{\rho_{1}}(b, t), t\right) .
\end{aligned}
$$

Assume that two level curves $\phi_{\rho_{1}}$ and $\phi_{\rho_{2}}$ collapse when $t$ tends to $T^{*}$ uniformly in $a \leq x_{1} \leq b$, i.e.

$$
\phi_{\rho_{2}}\left(x_{1}, t\right)-\phi_{\rho_{1}}\left(x_{1}, t\right) \sim \frac{1}{b-a} \int_{a}^{b}\left[\phi_{\rho_{2}}\left(x_{1}, t\right)-\phi_{\rho_{1}}\left(x_{1}, t\right)\right] d x_{1} .
$$

In other words, the distance between two level sets are comparable for $a \leq x_{1} \leq b$.

Let

$$
\delta\left(x_{1}, t\right)=\left|\phi_{\rho_{2}}\left(x_{1}, t\right)-\phi_{\rho_{1}}\left(x_{1}, t\right)\right|
$$

be the thickness of the front.

We define semi-uniform collapse on a curve if (2.1) and (2.2) holds and there exists a constant $c$, independent of $t$, such that

$$
\min \delta\left(x_{1}, t\right) \geq c \cdot \max \delta\left(x_{1}, t\right)
$$

for $a \leq x_{1} \leq b$, and for all $t \in\left[0, T^{*}\right)$.

We call the length $b-a$ of the interval $[a, b]$ the length of the front. 
Now we can state the following theorem.

Theorem 2.1. For a $Q G$ solution with a semi-uniform front, the thickness $\delta(t)$ satisfies

$$
\delta(t)>e^{-e^{A t+B}} \quad \text { for all } t \in\left[0, T^{*}\right)
$$

Here, the constants $A$ and $B$ may be taken to depend only on the length of the front, the semi-uniformity constant, the initial thickness $\delta(0)$, and the norm of the initial datum $\theta_{0}(x)$ in $L^{1} \cap L^{\infty}$.

Proof. From (9) we have

$$
\left|\frac{d}{d t} A(t)\right|<\frac{C}{b-a} \sup _{a \leq x_{1} \leq b}\left|\psi\left(x_{1}, \phi_{\rho_{2}}\left(x_{1}, t\right), t\right)-\psi\left(x_{1}, \phi_{\rho_{2}}\left(x_{1}, t\right), t\right)\right|
$$

where

$$
A(t)=\frac{1}{b-a} \int_{a}^{b}\left[\phi_{\rho_{2}}\left(x_{1}, t\right)-\phi_{\rho_{1}}\left(x_{1}, t\right)\right] d x_{1},
$$

and $C$ is determined by the semi-uniformity constant $c$.

The estimate of the difference of the value of the stream function at two different points that are close to each other is obtained by writing the stream function as follows:

$$
\psi(x, t)=-\int_{\Omega} \frac{\theta(x+y, t)}{|y|} d y
$$

and this is because $\psi=\left(-\triangle_{x}\right)^{-\frac{1}{2}} \theta$.

Therefore

$$
\begin{aligned}
\psi\left(z_{1}, t\right)-\psi\left(z_{2}, t\right) & =\int_{\Omega} \theta(y)\left(\frac{1}{\left|y-z_{1}\right|}-\frac{1}{\left|y-z_{2}\right|}\right) d y \\
& =\int_{\left|y-z_{1}\right| \leq 2 \tau}+\int_{2 \tau<\left|y-z_{2}\right| \leq k}+\int_{k<\left|y-z_{1}\right|} \\
& \equiv I_{1}+I_{2}+I_{3}
\end{aligned}
$$

where $\tau=\left|z_{1}-z_{2}\right|$.

Furthermore

$$
\begin{aligned}
\left|I_{1}\right| & \leq\|\theta\|_{L^{\infty}} \cdot \int_{\left|y-z_{1}\right| \leq 2 \tau}\left(\frac{1}{\left|y-z_{1}\right|}+\frac{1}{\left|y-z_{2}\right|}\right) d y \\
& \leq C \tau .
\end{aligned}
$$

We define $s$ to be a point in the line between $z_{1}$ and $z_{2}$; then $\left|y-z_{1}\right| \leq 2|y-s|$ and $I_{2}$ can be estimated by

$$
\begin{aligned}
\left|I_{2}\right| & \leq C \tau \cdot \int_{2 \tau<\left|y-z_{1}\right| \leq k} \max _{s}\left|\nabla\left(\frac{1}{|y-s|}\right)\right| d y \\
& \leq C \tau \cdot \int_{2 \tau<\left|y-z_{1}\right| \leq k} \max _{s} \frac{1}{|y-s|^{2}} d y \\
& \leq C \tau \cdot|\log \tau| .
\end{aligned}
$$


We use the conservation of energy to estimate $I_{3}$ by

$$
\left|I_{3}\right| \leq C \cdot \tau
$$

Finally, by choosing $\tau=\left|z_{1}-z_{2}\right|$ we obtain

$$
\left|\psi\left(z_{1}, t\right)-\psi\left(z_{2}, t\right)\right| \leq M\left|z_{1}-z_{2}\right||\log | z_{1}-z_{2}||
$$

where $M$ is a constant that depends on the initial data $\theta_{0}$. (See details in [3].)

Then we have

$$
\begin{aligned}
\left|\frac{d}{d t} A(t)\right| & \leq \frac{M}{b-a} \sup _{a \leq x_{1} \leq b}\left|\phi_{\rho_{2}}\left(x_{1}, t\right)-\phi_{\rho_{1}}\left(x_{1}, t\right)\left\|\log \mid \phi_{\rho_{2}}\left(x_{1}, t\right)-\phi_{\rho_{1}}\left(x_{1}, t\right)\right\|\right. \\
& \leq \frac{C \cdot M}{(b-a)}|A(t)||\log A(t)|
\end{aligned}
$$

and therefore

$$
A(t)>>A(0) e^{-e^{\frac{C \cdot M}{(b-a)} t}}
$$

Theorem 2.2. For a $2 D$ Euler solution with a semi-uniform front, the thickness $\delta(t)$ satisfies

$$
\delta(t)>e^{-[A t+B]} \text { for all } t \in\left[0, T^{*}\right) .
$$

Here, the constants $A$ and $B$ may be taken to depend only on the length of the front, the semi-uniformity constant, the initial thickness $\delta(0)$, and the norm of the initial vorticity in $L^{1} \cap L^{\infty}$.

The proof of Theorem 2.2 is similar to that of Theorem 2.1 with the difference that instead of the estimate (2.9), we have

$$
\left|\psi\left(z_{1}, t\right)-\psi\left(z_{2}, t\right)\right| \leq M\left|z_{1}-z_{2}\right|
$$

where $M$ is a constant that depends on the initial data $u_{0}$. (See details in [3].)

A consequence of Theorem 2.2 is that it rules out an exponential growth on the derivatives of the velocity for $2 \mathrm{D}$ Euler equations by a semi-uniform front. Similar estimates can be obtained for the 2D ideal Magneto-hydrodynamics (MHD) Equation, with the extra assumption that $\int_{0}^{T^{*}}|u|_{L^{\infty}}(s) d s$ is bounded up to the time of the blow-up. These estimates are a consequence of applying the mean value theorem in (2.8). Nevertheless in the case of MHD these estimates improve the results obtained in [6].

\section{REFERENCES}

1. P. Constantin, A. J. Majda, and E. Tabak. Formation of strong fronts in the 2-D quasigeostrophic thermal active scalar. Nonlinearity, 7:1495-1533, 1994. MR 95i:76107

2. P. Constantin, Q. Nie, and N. Schorghofer. Nonsingular surface-quasi-geostrophic flow. Phys. Lett. A, 24:168-172, 1998. MR 99a:76031

3. D. Cordoba. Nonexistence of simple hyperbolic blow-up for the quasi-geostrophic equation. Ann. of Math., 148 (3):1135-1152, 1998. MR 2000j:76020

4. D. Cordoba and C. Fefferman. Behavior of several 2D fluid equations in singular scenarios. Proc. Nat. Acad. Sci. USA, 98:4311-4312, 2001. CMP 2001:10

5. D. Cordoba and C. Fefferman. Scalars convected by a $2 D$ incompressible flow. Comm. Pure Appl. Math., 55 (2):255-260, 2001. CMP 2002:04

6. D. Cordoba and C. Marliani. Evolution of current sheets and regularity of ideal incompressible magnetic fluids in 2D. Comm. Pure Appl. Math., 53 (4):512-524, 2000. MR 2000i:76130 
7. A. Majda and E. Tabak. A two-dimensional model for quasi-geostrophic flow: comparison with the two-dimensional Euler flow. Physica D, 98:515-522, 1996. MR 97g:86005

8. K. Ohkitani and M. Yamada. Inviscid and inviscid-limit behavior of a surface quasigeostrophic flow. Phys. Fluids, 9:876-882, 1997. MR 97m:76032

Department of Mathematics, University of Chicago, Chicago, Illinois 60637

Current address: Department of Mathematics, Princeton University, Princeton, New Jersey 08540

E-mail address: dcg@math.princeton.edu

Department of Mathematics, Princeton University, Princeton, New Jersey 08540

E-mail address: cf@math.princeton.edu 